\title{
Giardiasis in endoscopy patients: A comparison of diagnostic techniques
}

DOONE R WATSON, DVM, MSc, Lloyd SutherLAND, MD, FRCPC, A DEAN BEFus, PHD

\begin{abstract}
The results of a study comparing techniques for diagnosis of giardiasis during endoscopy are presented. Methods of diagnosis included examination of duodenal biopsy impression smears, culture of both biopsies and aspirates, and examination of preserved aspirate. Giardiasis was diagnosed in three of 80 patients; in each case the impression smear was positive. Cultures of duodenal biopsies were positive in two patients; in the third patient a biopsy was not available. The preserved aspirate was positive in only one patient, while the aspirate cultures were never positive. It is proposed that during diagnostic endoscopy, routine submission of a duodenal biopsy impression smear from patients with unexplained small bowel diarrhea or abdominal discomfort is appropriate. Can J Gastroenterol 1990;4(1):1-4
\end{abstract}

Key Words: Diagnosis, Endoscopy, Giardia

\section{La giardiase mise en évidence par l'endoscopie: Comparaison des démarches diagnostiques}

RESUME: Les résultats d'une étude comparant les techniques endoscopiques utilisées dans le diagnostic d'infection à giardia sont présentés. Ces méthodes incluent l'examen des biopsies duodénales prélevées par empreinte directe, la culture des biopsies et des échantillons prélevés par aspiration, et l'examen des échantillons obtenus par aspiration et préservés. La giardiase a été diagnostiquée chez trois patients sur 80 . Pour chacun d'eux, la biopsie par empreinte directe était positive. Les cultures des biopsies duodénales étaient positives chez deux patients; aucune biopsie n'avait été fournie dans le cas du troisième. L'échantillon préservé n'était positif que chez un seul patient, alors que les cultures des prélèvements par aspiration n'étaient jamais positives. On propose qu'au cours de l'endoscopie diagnostique, une biopsie duodénale par empreinte directe soit systématiquement effectuée pour les patients souffrant de diarrhée de l'intestin grêle et de malaise abdominal inexpliqués.

Faculty of Medicine, University of Calgary, Calgary, Alberta

Correspondence and reprints: Dr Doone Watson, Department of Microbiology and Infectious Diseases, Faculty of Medicine, University of Calgary, 3330 Hospital Drive NW, Calgary, Alberta T2N 4N1. Telephone (403) 220-5065

Received for publication August 3, 1989. Accepted December 18, 1989 IARDIA LAMBLIA IS THE MOST
commonly isolated intestinal protozoan parasite in North America (1). Infected individuals may be asymptomatic or may exhibit a spectrum of disease severity. The clinical presentation ranges from a mild, self-limiting enteritis with complete recovery, to a chronic malabsorption syndrome $(1,2)$. The majority of cases of giardiasis are diagnosed by routine ova and parasite screening of stool, a rapid and inexpensive test. A negative result does not invariably exclude giardiasis as an infected patient does not always pass cysts, even when symptomatic (3). When dealing with a patient who has gastrointestinal symptoms suggestive of giardiasis but a negative stool exam, the clinician is faced with a dilemma - is additional testing (often expensive and time-consuming) needed to exclude the possibility of giardial infection?

Many gastroenterologists include endoscopic examination of the small bowel in their work-up of patients with unexplained gastrointestinal disorders. Thus, to assess techniques for identifying $\mathrm{G}$ lamblia infection in such patients the authors compared various techniques available during endoscopy. Duodenal aspirates were obtained 
during endoscopy and examined for trophozoites (4). Histological examination and impression smears of duodenal biopsies were assessed for trophozoites (5). As well, in accordance with the recent results of Gordts et al (6), cultures were employed to identify trophozoites from intestinal aspirates.

Since cultures of duodenal aspirates or biopsies are considered to be the most sensitive of these tests (6), they were compared to the examination of preserved samples of duodenal aspirates and impression smears of duodenal biopsies. In this paper the results are presented of a prospective study of giardiasis in 80 patients undergoing endoscopy for various gastrointestinal symptoms compatible with giardiasis.

\section{PATIENTS AND METHODS}

Study description: Eighty patients with unexplained diarrhea, abdominal discomfort, abdominal gas, malabsorption or weight loss, who required an upper endoscopy as part of their gastrointestinal assessment, were included in the study. All procedures were performed in the Day Care Endoscopy Unit at the Foothills Hospital in Calgary, Alberta. Patients undergoing repeat endoscopies for follow-up of gastric ulcers were excluded from the study, as were patients with diseases of known peptic and hepatic origin.

The study population consisted of 36 women and 44 men, aged 23 to 68 years (mean age 41.3 years). As a result of an ongoing study of AIDS at the Foothills Hospital there was a high percentage $(15 \%)$ of HIV-positive patients. Giardiasis had not been diagnosed in any of the patients at the time of endoscopy, although the extent of assessment for giardiasis was variable.

The endoscopy procedure required overnight fasting, followed by sedation with meperidine and benzodiazepine. Endoscopy was carried out in standard fashion using an Olympus panendoscope. The scope was advanced into the second stage of the duodenum where samples were taken for each of the four diagnostic techniques under assessment. Twenty millilitres of sterile saline were injected into the biopsy channel of the endoscope and 5 to 10 $\mathrm{mL}$ of duodenal aspirate were withdrawn by suction into a sterile disposable suction trap. Since the saline solution originally in use contained bactericidal components, it was replaced with a preservative-free saline to minimize difficulties with culture of trophozoites. One millilitre of the aspirate was removed aseptically and placed in a tube of culture media (TYIS-33 media supplemented with $10 \%$ CLEX, NCTC 109 modified vitamin mix and antibiotics) (6). Since most antibiotics have no effect on the growth of giardia in culture (7), the following antibiotics were added to the culture media: penicillin $300 \mathrm{U} / \mathrm{mL}$, streptomycin $300 \mu \mathrm{g} / \mathrm{mL}$, gentamycin $40 \mu \mathrm{g} / \mathrm{mL}$, tobramycin $100 \mu \mathrm{g} / \mathrm{mL}$, amphotericin $1 \mu \mathrm{g} / \mathrm{mL}$. SAF preservative (15 g sodium acetate, $20 \mathrm{~mL}$ glacial acetic acid, $40 \mathrm{~mL} 40 \%$ formaldehyde, $925 \mathrm{~mL}$ deionized water) was added to the remaining aspirate to make a one in four dilution of duodenal fluid to preservative.

Finally, three duodenal biopsies were obtained using standard endoscopy biopsy forceps. The first biopsy was used to make an imprint smear ensuring that the mucosal surface was smeared rather than touched against the glass slide. The slide was allowed to air dry. The second biopsy was placed in a tube of culture media and the third biopsy in $10 \%$ formalin and sent for routine histopathology. The preserved aspirate and impression smear were sent to the microbiology laboratory for standard examination for parasites, and were examined within 2 to $4 \mathrm{~h}$ by one of three technicians. The two culture tubes were incubated at $37^{\circ} \mathrm{C}$ until examination. They were examined for trophozoites under a Zeiss inverted microscope $(x 400)$ within $2 \mathrm{~h}$ of collection. The culture tubes were maintained at $37^{\circ} \mathrm{C}$ for 10 days and examined daily for trophozoites by the same individual.

The study was designed to assess procedures already in practice, so no attempts were made to alter the standard protocol for the collection or examination of aspirates and biopsies. The only new addition to the protocol was the collection of an additional aspirate and biopsy for culture.

\section{RESULTS}

Giardiasis was diagnosed in three of 80 patients examined. In each case the biopsy impression smear was positive. Cultures of duodenal biopsies were positive in two patients. In the third patient a biopsy for culture was not available. The preserved aspirate was positive in only one of the three positive patients, while the aspirate cul. tures were never positive. Statistical analysis was not performed because of the low attack rate and the sample size. Routine histological examination of duodenal biopsies revealed trophozoites in only one of the three patients.

The results of the study reflected the difficulty of obtaining standardized samples in a busy referral facility. Stool samples were rarely submitted at the time of endoscopy. This is not an unusual problem considering the preparation of patients for endoscopy (laxatives, enemas and/or fasting). In addition, the procedures which required more time, such as aspirates, were excluded in several patients who did not tolerate the procedure well. The culture procedure required thawing of the culture media 10 mins prior to use. In two or three cases this was not done and culturing was delayed. Staff changes led to a problem in one case in which culture samples were not collected. Unfortunately, this patient was one of the few positive for giardiasis.

The first positive case was a patient with vague abdominal discomfort and mild duodenitis. Trophozoites were seen in the impression smear, the biopsy culture tube and the biopsy examined histologically. There was no evidence of trophozoites in either the preserved or cultured aspirate. An axenic in vitro culture of this isolate was established and named C-1. Subsequent stool examination revealed large numbers of giardia cysts. The patient was treated with metronidazole (250 $\mathrm{mg}$ tid) for 10 days and returned one month later with persistent symptoms. Endoscopy was repeated and once again trophozoites were seen in the biopsy impression smear and biopsy 
culture. Both aspirate samples were negative. A second axenic culture was established named C-2.

The second case was an HIV-positive patient with mild diarrhea. Both biopsy samples were positive while the aspirates were negative. The authors were unable to establish an axenic culture. Stool samples were not available at the time of endoscopy, but earlier stool samples had been negative. No trophozoites were observed by histology. A third case of giardiasis was seen in the endoscopy unit during this study. Both impression smear and preserved aspirate were positive, but samples were not available for culture. Histological examination did not reveal trophozoites.

Despite the presence of antibiotics in the culture media, there was a persistent problem with bacterial and fungal overgrowth. This was most noticeable in the aspirate cultures, often within $24 \mathrm{~h}$. Yeast contamination occurred within $48 \mathrm{~h}$ in eight of 12 aspirate cultures from HIV-positive patients despite the use of amphotericin.

\section{DISCUSSION}

Considering the large numbers of reported cases of giardiasis in Alberta (1613 in 1986 of which 620 were from Calgary) (personal communication), the authors were surprised at the low prevalence $(3.75 \%)$ of giardiasis in the endoscopy patients with various gastrointestinal problems compatible with giardiasis. Previous studies in the United States and Australia have shown prevalences ranging from 0.7 to $15.5 \%$ (8-10). Known HIV-positive patients were included in this study because previous results (11) indicated that these patients do not have a greater incidence of giardiasis than HIV-positive individuals.

There are several explanations for the low rate of positivity in this study. The majority of cases of giardiasis are acute and diagnosed by stool examination, and would therefore not be seen in a referral clinic. There is also an age bias, in that the majority of infections are found in the pediatric population which is seen elsewhere, at the Alberta Children's Hospital. In Alberta almost $50 \%$ of reported cases of giardiasis occur in patients under the age of 15 years (12).

Nevertheless, the present results indicate that a small percentage of patients undergoing endoscopy are infected with G lamblia, and an appropriate diagnosis may be missed if biopsies are not taken. Prior to this study the technique most commonly used by the endoscopy unit for detection of $\mathrm{G}$ lamblia was examination of duodenal aspirates. Other methods were occasionally used, including impression smears and histological examination. The authors showed that two of the three positive cases would not have been detected if duodenal aspirates had been the sole specimens submitted. These results differ from those of other investigators who suggested that examination of duodenal aspirates was as sensitive as mucosal impression smears $(9,13)$. Kerlin et al (9) noted in their study that all 21 patients with positive impression smears had positive duodenal aspirates. They used a polyethylene catheter to obtain the aspirates, rather than the endoscope the present authors used. Gordts et al (13) also found that all of their patients positive on impression smear had positive duodenal aspirates, using a pediatric Watson tube modified with a double lumen tube and monitored by radioscopy.

Kamath et al (14) compared four methods for detecting giardia, namely: stool exam, duodenal aspirates, jejunal biopsies and impression smears of jejunal mucosa. They acknowledged, as found here, that the impression smear was the most sensitive and easily read test. However, they promoted the use of the aspirate because it was almost as sensitive and less invasive. In their study, 12 patients were positive on smear and biopsy, while 10 were positive on aspirate. The aspirates were obtained using a modified Crosby capsule and fluoroscopy.

There are several reasons that could explain the present low rate of positive aspirates by both direct examination and culture. The collection of duodenal aspirates through the biopsy channel of the endoscope involved several steps which might have influenced the sen- sitivity of the test. Obtaining aspirates without saline irrigation was difficult and time-consuming; thus many of the samples obtained had been diluted with saline. As a result the precise amount of intestinal fluid in the sample could not be determined and negative results may have reflected inadequate sampling. The endoscope was cleaned between cases with soap and disinfectants and, although it was rinsed with distilled water prior to use, it is possible that enough disinfectant remained to decrease trophozoite viability.

In addition, the aspirates sent to the laboratory for direct examination were preserved, since trophozoites are fragile organisms and may die and break up if not examined immediately. Intestinal fluid is also known to contain components toxic to trophozoites (15). The major difficulty with this approach is that trophozoite motility cannot be used to facilitate their detection.

It would appear that the collection of duodenal aspirates would be more effective if a modified Crosby capsule or feeding tube was used as described earlier $(13,14)$, although this would require fluoroscopy which may not be available. The use of a small polyethylene catheter inserted in the suction channel may improve the collection technique.

In contrast to the collection of aspirates, the collection of duodenal biopsies is a straightforward procedure easily accomplished during routine endoscopic examination. The most important requirement of preparing the impression smear is that the mucosal surface be smeared on the slide. It is not surprising that biopsy cultures had a higher success rate when one considers the biology of the parasite. G lamblia burrows into the mucus lining the epithelium and attaches to the brush border, which protects it from the toxic intestinal contents. When a biopsy is taken the parasites remain in a protected environment, and are more likely to be established in culture.

Recently, a sensitive test for detection of giardia antigen in stool has been developed, which has been shown to detect up to $30 \%$ more positive samples than routine ova and parasite screens 
(16). This test is not designed to replace ova and parasite screens as it is more expensive (approximately double the cost), but it may significantly increase the sensitivity of giardiasis testing. For various reasons, including economics and limited availability of the kit, this test is still not in widespread use; nevertheless, it may become a useful tool in the diagnosis of these difficult cases of giardiasis.

ACKNOWLEDGEMENTS: The authors are grateful to Jessie Darling, Joan Craig and the gastroenterologists in the endoscopy unit for their help with this study. They also thank Dr D Church and Bev Madden of the Foothills Hospital Microbiology Department for their cooperation. The assistance of the Gastrointestinal Research Group, University of Calgary was appreciated. This study was supported by the Alberta Heritage Foundation for Medical Research and the Medical Research Council of Canada.

\section{REFERENCES}

1. Meyer EA, Jarroll EL. Giardiasis. Am J Epidemiol 1980;111:1-12.

2. Pickering LK, Engelkirk PG. Giardia lamblia. Pediatr Clin North Am 1988;25:565-77.

3. Wolfe MS. Giardiasis. N Engl J Med 1978;298:319.

4. Sealy DP, Schuman SH. Giardiasis: A common and underrecognized enteric pathogen. J Fam Pract 1981;12:47-54.

5. Deckelbaum RJ, Korman SH. Giardia lamblia: New outlooks. Front
It was unfortunate that stool samples were unavailable at the time of endoscopy for most of the patients. However, as previously discussed, this is not an uncommon problem in referral clinics. It is often easy to optimize test conditions in a research laboratory and obtain good results; however, compliance and ease of performing the tests in a hospital setting drastically influence the sensitivity of diagnostic tests.

Gastrointest Res 1986;13:387-97.

6. Gordts B, Retore P, Cadranel S, Hemelhof W, Rahman M, Butzler ]. Routine culture of Giardia lamblia trophozoites from human duodenal aspirates. Lancet 1984;ii:137-8.

7. Jakubowski W, Meyer EA, Nash TE, Hibler CP. Panel discussion on methods of handling Giardia in the laboratory. In: Wallis PM, Hammond BR, eds. Advances in Giardia Research. Calgary: University of Calgary Press, 1988:291-4.

8. McHenry R, Bartlett MS, Lehman GA, O'Connor KW. The yield of routine duodenal aspiration for Giardia lamblia during esophagogastroduodenoscopy. Gastrointest Endosc 1987;33:425-6.

9. Kerlin P, Ratnaike RN, Butler R, Gehling N, Grant AK. Prevalence of giardiasis: A study at upper-gastrointestinal endoscopy. Dig Dis 1978;23:940. 2.

10. Carr MF, Ma J, Green PHR. Giardia lamblia in patients undergoing endoscopy: Lack of evidence for a role in nonulcer dyspepsia. Gastroenterology 1988;95:972-4.
Based on this study the authors sug. gest that the routine submission of a duodenal biopsy impression smear from all patients with small bowel diarrhea, malabsorption or weight loss is warranted in areas with endemic giardiasis. The procedure is easily performed and does not add significantly to the overall cost of the investigation (currently the additional cost is under five dollars, less than $5 \%$ of the total cost).

11. Janoff EN, Smith PD, Blaser MJ. Acute antibody responses to Giardia lamblia are depressed in patients with AIDS. J Infect Dis 1988;157:798-804.

12. Waters J. Selected notifiable disease statistics. Epidemiol Notes Rep. 1988;12(2).

13. Gordts B, Hemelhof W, van Tilborgh K, Retore P, Cadranel S, Butzler J. Evaluation of a new method for routine in vitro cultivation of Giardia lamblia trophozoites from human duodenal fluid. J Clin Microbiol 1985;22:702-4.

14. Kamath KR, Murugasu R. A comparative study of four methods for detecting Giardia lamblia in children with diarrheal disease and malabsorption. Gastroenterology 1974;66:16-21.

15. Das S, Reiner DS, Zenian J, et al. Killing of Giardia lamblia trophozoites by human intestinal fluid in vitro. J Infect Dis 1988;157:1257-60.

16. Rosoff JD, Sanders CA, Sonnad SS, etal. Stool diagnosis of giardiasis using a commercially available enzyme immunoassay to detect Giardia-specific antigen 65 (GSA 65). J Clin Microbiol 1989;27:1997-2002. 


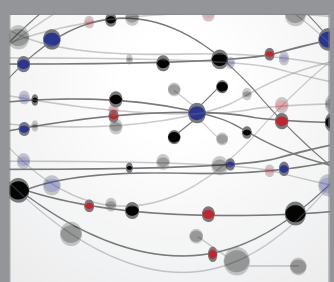

The Scientific World Journal
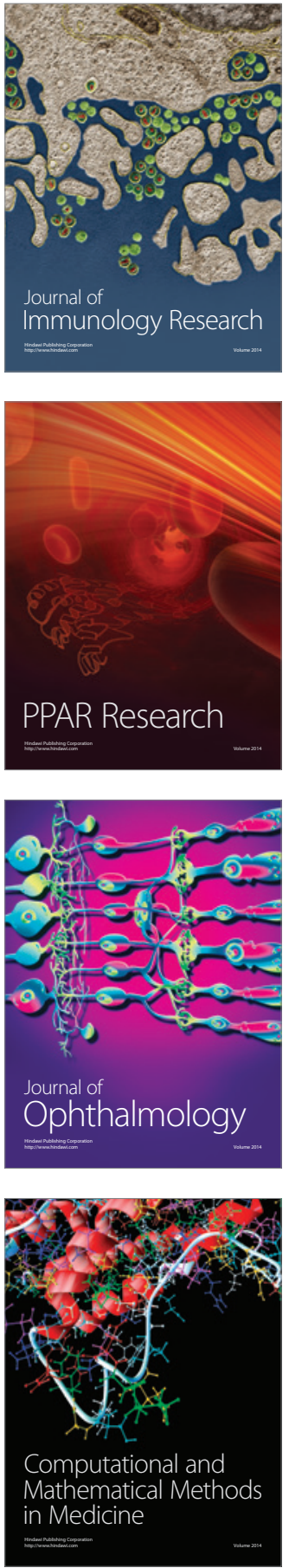

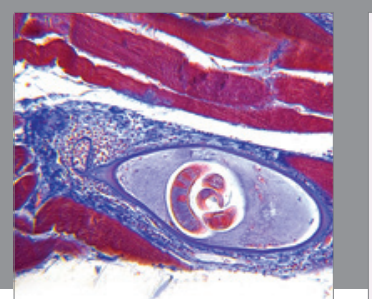

Gastroenterology Research and Practice

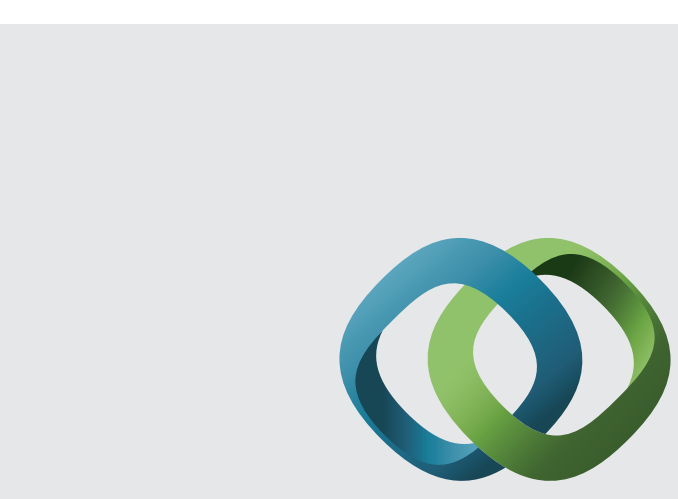

\section{Hindawi}

Submit your manuscripts at

http://www.hindawi.com
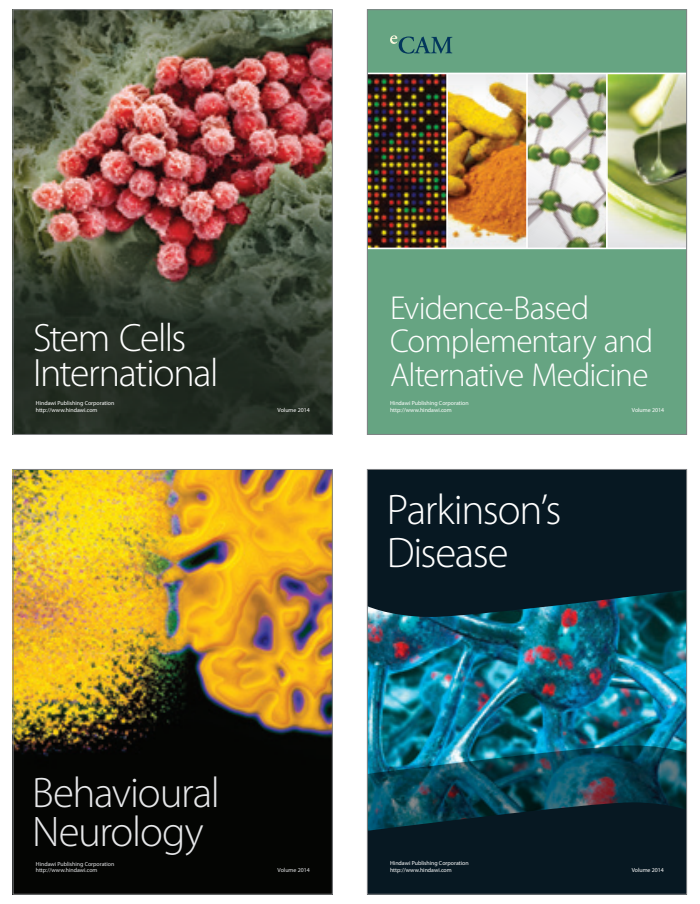
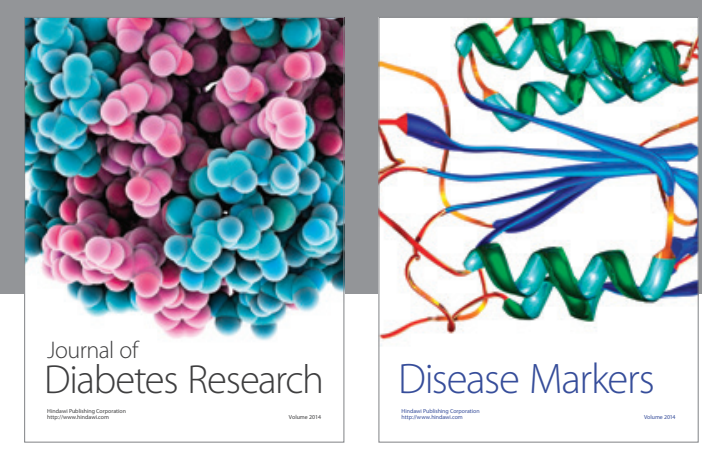

Disease Markers
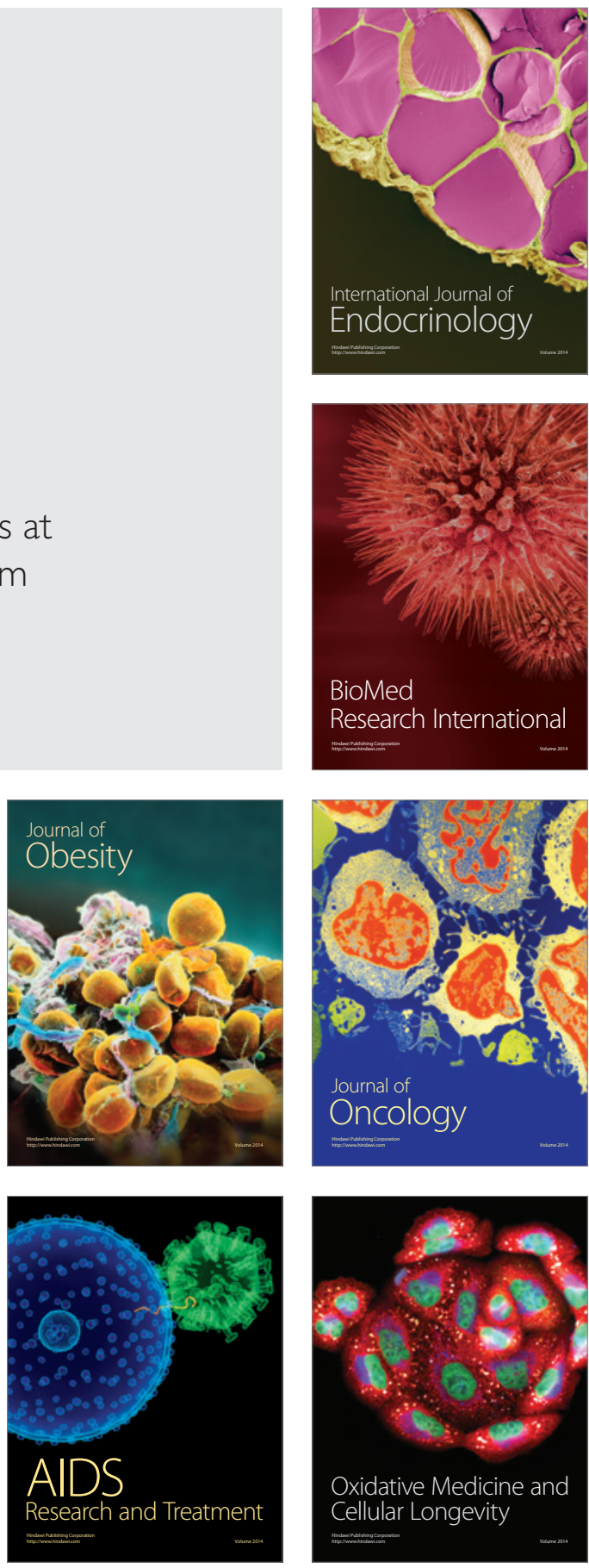